\title{
Bead Capture and Release on a Magnetic Sensor in a Microfluidic System
}

Dalslet, Bjarke Thomas; Damsgaard, Christian Danvad; Freitas, S.C.; Freitas, P.P.; Hansen, Mikkel Fougt

Link to article, DOI:

10.1109/ICSENS.2008.4716426

Publication date:

2008

Document Version

Publisher's PDF, also known as Version of record

Link back to DTU Orbit

Citation $(A P A)$ :

Dalslet, B. T., Damsgaard, C. D., Freitas, S. C., Freitas, P. P., \& Hansen, M. F. (2008). Bead Capture and Release on a Magnetic Sensor in a Microfluidic System. Poster session presented at 2008 IEEE Sensors, Lecce, Italy. https://doi.org/10.1109/ICSENS.2008.4716426

\section{General rights}

Copyright and moral rights for the publications made accessible in the public portal are retained by the authors and/or other copyright owners and it is a condition of accessing publications that users recognise and abide by the legal requirements associated with these rights.

- Users may download and print one copy of any publication from the public portal for the purpose of private study or research.

- You may not further distribute the material or use it for any profit-making activity or commercial gain

- You may freely distribute the URL identifying the publication in the public portal 


\title{
Bead Capture and Release on a Magnetic Sensor in a Microfluidic System
}

\author{
Bjarke T. Dalslet*, Christian D. Damsgaard*, Susana C. Freitas ${ }^{\dagger}$ Paulo P. Freitas ${ }^{\dagger}$ \\ and Mikkel F. Hansen* \\ *Department of Micro and Nanotechnology, Technical University of Denmark \\ DTU Nanotech, Building 345 East, DK-2800 Kongens Lyngby Denmark \\ Email: bjarke.dalslet@nanotech.dtu.dk \\ ${ }^{\dagger}$ INESC-microsystems and nanotechnologies, Rue Alves Redol, 9-1, 1000-029 \\ and Instituto Superior Tecnico, Physics Department, Avenida Rovisco Pais, 1000 Lisboa, Portugal
}

\begin{abstract}
Planar Hall effect magnetic sensors for detection of biological agents using surface treated magnetic beads are integrated with a fluid injection system. The response of the sensors is used to evaluate bead capture rates for different bead concentrations $c$ and fluid flow rates $Q$, and to monitor subsequent removal of the beads. It is found that the capture rate scales directly with $c$ and that it depends linearly on $Q$. At low $Q$ the capture rate is only partially due to gravitational sedimentation of beads. At higher $Q$ an additional term proportional to $Q$ becomes important, which is attributed to capture of beads by the magnetic fields near the sensor. It is shown that beads can be washed off the sensor surface.
\end{abstract}

\section{INTRODUCTION}

Exchange biased permalloy planar Hall effect sensors have been developed for biosensor applications [1], [2]. Magnetic biosensors rely on the sensitivity to the magnetic field of magnetic beads attached to the sensor via an appropriate bioassay. An understanding of the dynamics and mechanisms of bead capture on the sensor is important for optimizing incubation and washing conditions. To ensure reproducible reaction and washing conditions and also to enable downstream biosensing, the magnetic biosensor is integrated in a fluidic system. The sensor signal is monitored while inert bead suspensions with a range of concentrations are injected at various flow rates. This allows us to investigate the dynamics of the beads sedimenting on the sensors. The subsequent washing step to remove the unspecifically bound beads is also monitored.

\section{THEORY}

The sensors consist of a layer of $\mathrm{Ni}_{81} \mathrm{Fe}_{19}$ exchange pinned by an antiferromagnetic layer of $\mathrm{Mn}_{74} \mathrm{Ir}_{26}$. They were fabricated with an easy magnetization direction in the positive $x$-direction as described in [1]. Fig. 1 shows the sensor layout on a chip and the stack cross-section. This sensor is sensitive to fields along the $y$-axis on the central $w \times w=40 \mu \mathrm{m} \times 40 \mu \mathrm{m}$ area. Upon application of a current $I_{x}$ in the $x$-direction, the planar Hall effect yields a potential difference in the $y$ direction, which for low fields is given by

$$
V_{y}=S_{0} H_{y} I_{x}
$$

where $S_{0}$ is the sensitivity and $H_{y}$ is the average magnetic field in the $y$-direction over the sensor [1]. The sign convention has been chosen such that a positive $H_{y}$ gives rise to a positive $V_{y}$. This field has a contribution $H_{\mathrm{y}, \mathrm{DC}}$ from any external DC magnetic fields and beads being magnetized by these external fields. The bias current in the sensor layers will induce another contribution to the magnetic field in the sensor, $\gamma_{0} I_{x}$. The magnetic field from the bias current will also magnetize any superparamagnetic beads in the vicinity, giving rise to yet another contribution to the magnetic field in the sensor, $\gamma_{1} I_{x}$. Here $\gamma_{0}$ is a constant depending on the stack and sensor geometry and $\gamma_{1}$ depends on the amount and distribution of magnetic beads on the sensor. Thus,

$$
H_{y}=H_{\mathrm{y}, \mathrm{DC}}+\left(\gamma_{0}+\gamma_{1}\right) I_{x} .
$$

Experimentally, an AC bias current $I_{x}=I_{x, 0} \sin (\omega t)$ will be applied and the response detected by lock-in technique. An analysis yields that the contribution to $V_{y}$ from $H_{\mathrm{y}, \mathrm{DC}}$ is detected in the $1^{\text {st }}$ harmonic in-phase susceptibility and that the self-field contribution is detected in the $2^{\text {nd }}$ harmonic outof-phase susceptibility. The latter is

$$
V_{y, 2}^{\prime \prime}=-\frac{1}{2 \sqrt{2}} S_{0}\left(\gamma_{0}+\gamma_{1}\right) I_{x, 0}^{2}
$$

where the prefactor comes from the lock-in method. $V_{y, 2}^{\prime \prime}$ will be the contribution discussed in this work. A bead placed on the middle of the sensor area will experience a magnetic field in the negative $y$-direction when a positive current $I_{x}$ is applied. Thus, it will give rise to a dipole field acting on the sensor in the positive $y$-direction, i.e., the presence of beads will give rise to a positive $\gamma_{1}$ and hence a negative change of $V_{y, 2}^{\prime \prime}$.

The response of the sensor to a certain coverage of magnetic beads can be calculated by first finding the magnetic field from the sensor acting on the beads and then calculate the average dipole field acting on the sensor from the beads [3]. When the beads are randomly distributed and much smaller than the dimension of the sensor area, the signal will be proportional to the bead coverage when this is less than one monolayer [3].

\section{EXPERIMENTAL}

A sketch of the sensor chip layout and the stack is shown in Fig. 1. The sensors are manufactured on a $\mathrm{Si}$ substrate 


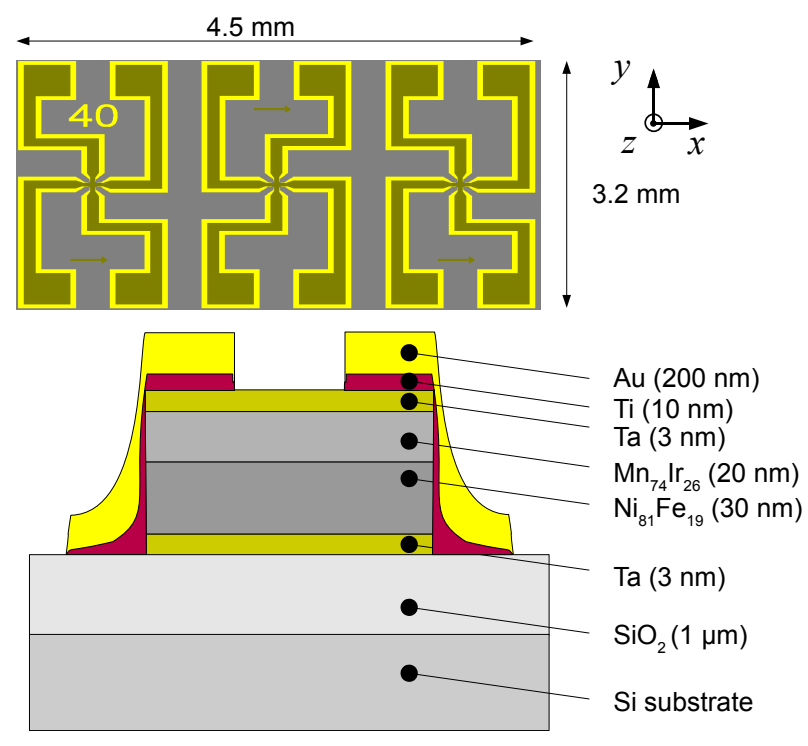

Fig. 1. Top view and schematic cross-sectional view of a chip.

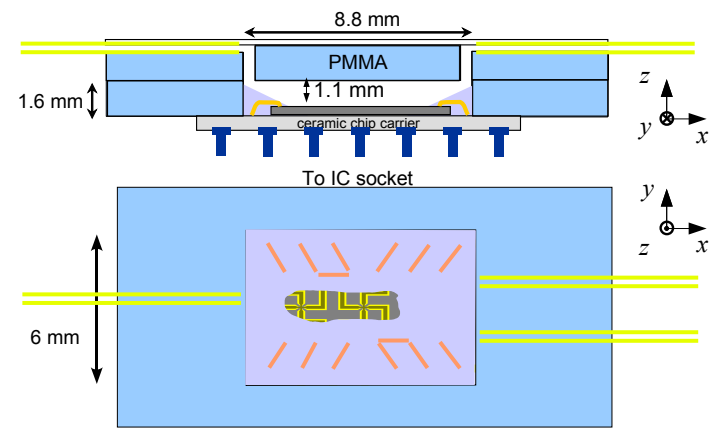

Fig. 2. Schematic cross-sectional and top views of the fluid system defined in PMMA and attached to the ceramic chip carrier.

with a $1 \mu \mathrm{m}$ thick thermal oxide. The sensor stack with a top-pinned active layer of $\mathrm{Ni}_{81} \mathrm{Fe}_{19}$ with $t_{\mathrm{FM}}=30 \mathrm{~nm}$ is deposited by ion beam deposition as described in [4] and then defined by ion milling. During deposition, a magnetic flux density of $4 \mathrm{mT}$ was applied to define the magnetic easy direction in the $x$-direction (see Fig. 1). The contacts are deposited by e-beam evaporation and defined by lift-off. A chip has three sensor crosses, each with an active area of $w \times w=40 \mu \mathrm{m} \times 40 \mu \mathrm{m}$. The sensitivity of the sensor, $S_{0}$, was measured to be $6.2 \cdot 10^{-5} \mathrm{~V} \mathrm{~m} \mathrm{~A}^{-2}$.

The chip is mounted on and wire bonded to a ceramic chip carrier. A fluid system is assembled around the sensor and substrate as sketched on Fig. 2. The fluid system is defined by laser ablation in PMMA and consists of a $2 \mathrm{~mm}$ thick fluid chamber plate glued to the ceramic chip carrier and a top with fluid inlets and outlet. The channels on the top part are sealed by lamination foil. The wire bonds are protected with silicone

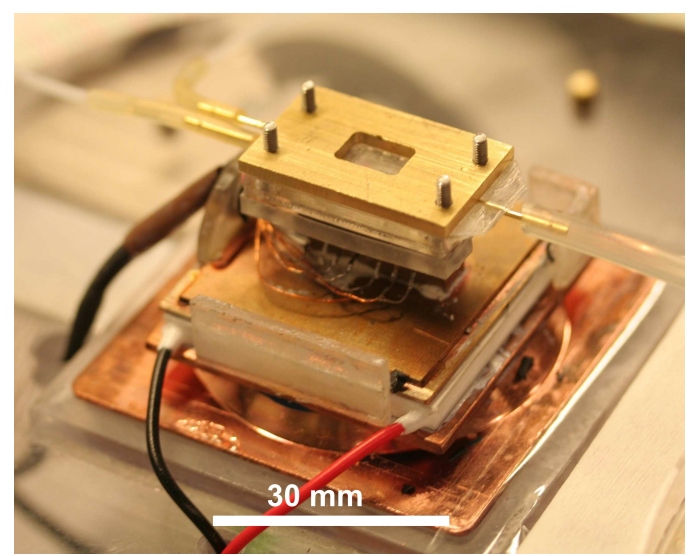

Fig. 3. The assembled fluid system on the Peltier element based temperature control system.

glue (Elastosil E41, Wacker Chemie, München, Germany). This glue also seals the interface between the ceramic chip carrier and the fluid chamber plate. The two-part fluid system is sealed by an O-ring. The channel cross section at the chip is $6 \mathrm{~mm} \times 1.1 \mathrm{~mm}$.

Two inlets allow the injection of a water suspension of Dynabeads MyOne magnetic beads and distilled water for cleaning. MyOne beads have a diameter of $1.05 \mu \mathrm{m}$, a density of $1800 \mathrm{~kg} / \mathrm{m}^{3}$ and a magnetic susceptibility of 1.46 . Two Harvard Apparatus PHD 2000 syringe pumps control the flow rates of the injected solutions. An AC sensor bias current with a frequency $2200 \mathrm{~Hz}$ is supplied by a Keithley 6221 current source. After preamplification in a Stanford Research Systems (SRS) SR552 bipolar preamplifier, the $2^{\text {nd }}$ harmonic out-ofphase susceptibility is extracted by a SRS SR830 lock-in amplifier. The system temperature is kept constant at $28^{\circ} \mathrm{C}$ by cooling/heating of a $\mathrm{Cu}$ block with an integrated thermometer by use of a Peltier element (see Fig. 3).

Before experiments, the fluid system was flushed with distilled water to remove air bubbles. The sensor bias current $I_{x, 0}=10 \mathrm{~mA}$ was on at all times. Then, a syringe with a bead solution of concentration $c$ was shaken to redisperse sedimented beads. Subsequently, the bead solution was injected at a flow rate $Q$ and the response of all three sensors was monitored vs. time. The time $t=0$ was defined as the instant when beads entered the fluid system and deviations from baseline sensor responses were observed. After monitoring the responses of the three sensors on the chip vs. time, the fluid system was flushed at a high $Q$ with distilled water to remove the beads. It was verified that the sensor signals returned to their initial level after washing. The three sensors on each chip gave similar results.

\section{RESUlts}

Figure 4 shows the sensor response vs. $t \cdot c$ for bead solutions with different concentrations, $c$, injected at $Q=0.83 \cdot 10^{-9}$ $\mathrm{m}^{3} / \mathrm{s}$. The sensor response is flat until $t=0$ when the bead solution enters the fluid system. The signal value then drops 


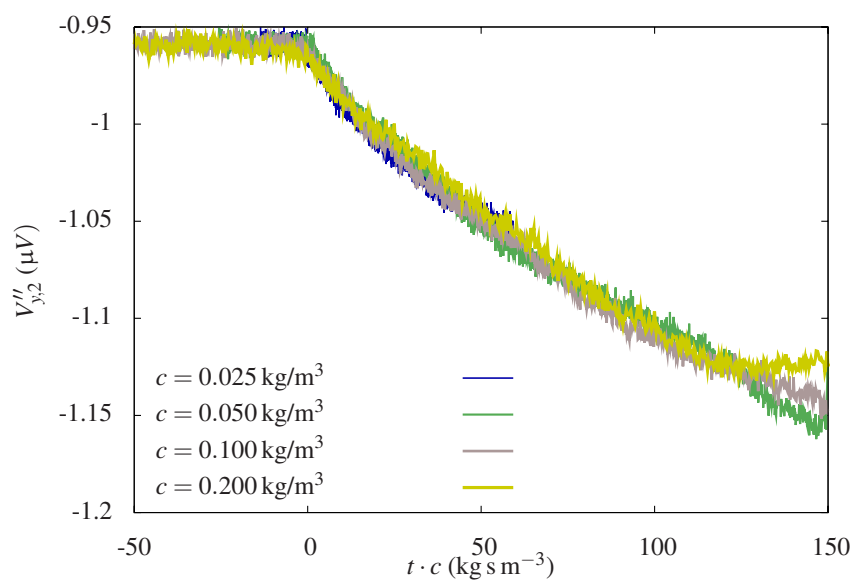

Fig. 4. Sensor response, $V_{y, 2}^{\prime \prime}$, as function of time, $t$, times the bead concentration, $c$ for experiments carried out with $Q=0.83 \cdot 10^{-9} \mathrm{~m}^{3} / \mathrm{s}$ and the indicated bead concentrations.

to more negative values as expected. The initial steep response from $c \cdot t=0$ to about $t \cdot c \approx 15 \mathrm{~kg} \mathrm{~s} \mathrm{~m}^{-3}$ in Fig. 4 is observed to be approximately linear in time, after which the change with time slows down. The initial linear response was observed to be reproducible in repeated experiments whereas the later response could vary from experiment to experiment. Thus, it is meaningful to define and discuss the initial slope of the sensor response, $S(c, Q) \equiv \frac{\partial V_{y, 2}^{\prime \prime}}{\partial t}$. The data in Fig. 4 show that, for the investigated bead concentrations and in the time intervals where $V_{y, 2}^{\prime \prime}$ depends linearly on the time, $S(c, Q)$ is proportional to $c \cdot t$ for a fixed value of $Q$. Thus, there does not seem to be a significant effect of fluid or magnetic bead-bead interactions.

Figure 5 shows the sensor response vs. $t$ for bead solutions with a fixed concentration of $c=0.2 \mathrm{~kg} / \mathrm{m}^{3}$ injected at various $Q$. Again, a flat response is observed until $t=0$ after which the response is approximately linear in time for a brief period. For long times, the signal is observed to level off and approach a steady state value. While the signal value at steady state does not vary systematically with $Q$, the time needed to reach a steady state is shorter when $Q$ is high. In the experiment with $Q=14 \cdot 10^{-9} \mathrm{~m}^{3} / \mathrm{s}$, the system was flushed with distilled water at $t=200 \mathrm{~s}$ which removed the beads and it is observed that the signal returns to its initial level.

Figure 6 shows $-S(c, Q)$ as a function of $Q$ extracted from a series of experiments with fixed $c$. It is found that $-S(c, Q)=a+b \cdot Q$, where $a$ and $b$ are positive constants.

Figure 7 shows the sensor response vs. time, $t$, after bead capture and during a subsequent exposure to a flow of distilled water with increasing values of $Q$ as indicated on the graph. We find that applying a flow of $Q=0.05 \cdot 10^{-9} \mathrm{~m}^{3} / \mathrm{s}$ will remove beads from the sensor, and that $Q=50 \cdot 10^{-9} \mathrm{~m}^{3} / \mathrm{s}$ is sufficient to reach the initial level of the sensor response indicating that no beads remain on the sensor.

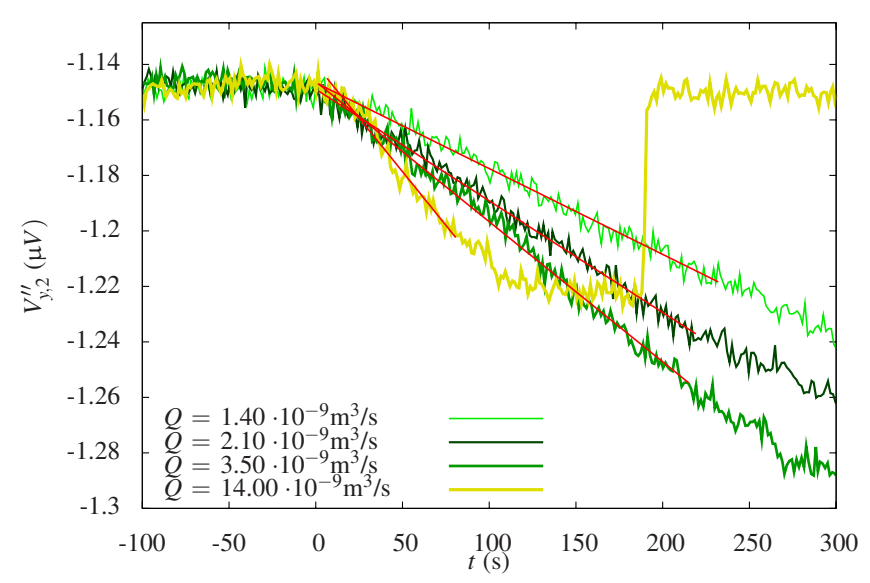

Fig. 5. Sensor response, $V_{y, 2}^{\prime \prime}$, as function of $t$ for experiments carried out with $c=0.2 \mathrm{~kg} / \mathrm{m}^{3}$ and the indicated $Q$. The lines indicate the linear fits of $V_{y, 2}^{\prime \prime}$ vs. $t$ used for extracting $S(c, Q)$.

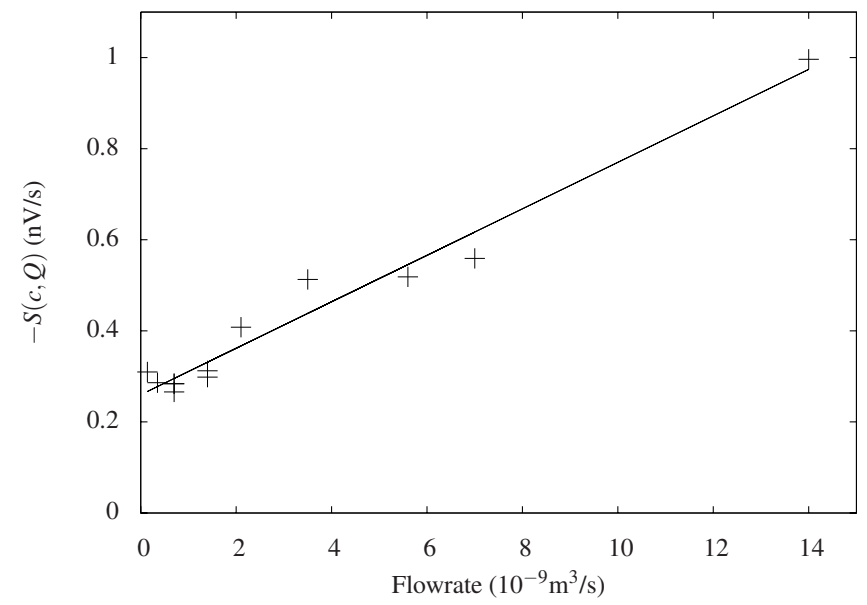

Fig. 6. Values of $-S(c, Q)$ as function of $Q$. The fit corresponds to $a=$ $0.26 \mathrm{nV} / \mathrm{s}$ and $b=0.051 \mathrm{nVm}^{-3}$.

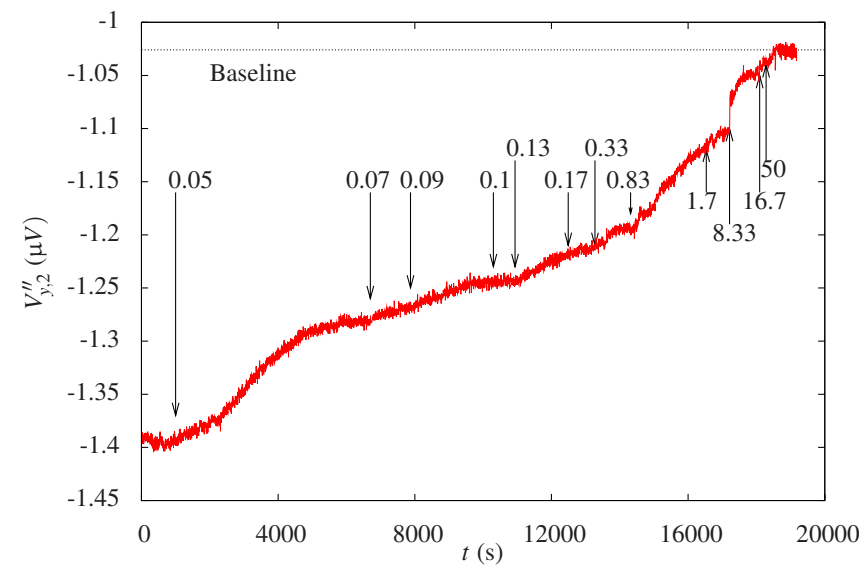

Fig. 7. Sensor response, $V_{y, 2}^{\prime \prime}$, as function of $t$. Numbers denote the applied $Q$ of water without beads (in $10^{-9} \mathrm{~m}^{3} / \mathrm{s}$ ). The horizontal line denotes the initial averaged sensor signal before it was exposed to the bead solution. 


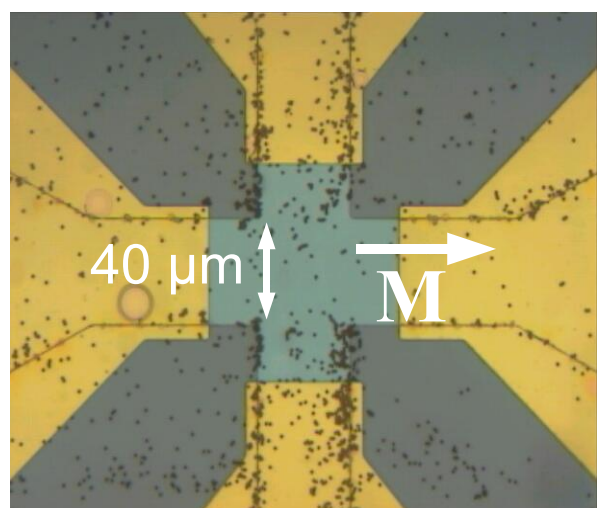

Fig. 8. Sensor after measurements. The black dots are beads deposited on the ridges of the permalloy stack, where the gradient of the magnetostatic field is high. The arrow indicates the magnetization direction of the ferromagnetic layer.

\section{Discussion}

We first consider the effect of gravitational sedimentation of the beads. Assuming that the fluid drag force on a single bead of radius $r$ is given by the Stokes drag, we find from Newton's second law that the MyOne beads sediment gravitationally with a velocity of

$$
v_{z}=\frac{2}{9} \frac{g\left(\rho_{\mathrm{b}}-\rho_{\mathrm{l}}\right) r^{2}}{\eta} \simeq-0.54 \mu \mathrm{m} / \mathrm{s}
$$

where $g=-9.82 \mathrm{~m} / \mathrm{s}^{2}$ is the gravitational acceleration, $\rho_{\mathrm{b}}$ and $\rho_{\mathrm{l}}$ are the densities of the beads and water, respectively, and $\eta$ is the viscosity.

The results in Fig. 5 and Fig. 6 show that a minimum value of $-S(c, Q)=0.26 \mathrm{nV} / \mathrm{s}$ exists for $Q<2 \cdot 10^{-9} \mathrm{~m}^{3} / \mathrm{s}$, corresponding to sedimentation in a negligible fluid flow. A close packed monolayer (ML) of MyOne beads contains $\frac{1}{2 \sqrt{3} r^{2}}=1.0 \cdot 10^{12}$ beads $/ \mathrm{m}^{2}$. The used bead concentration of $0.2 \mathrm{~kg} / \mathrm{m}^{3}$ corresponds to a bead number density of $1.8 \cdot 10^{14}$ beads $/ \mathrm{m}^{3}$. Thus, if all beads sediment at the velocity of 0.54 $\mu \mathrm{m} / \mathrm{s}$, the bead sedimentation rate is $9.9 \cdot 10^{-5} \mathrm{ML} / \mathrm{s}$. A calculation of the average dipole field from a close packed monolayer of MyOne beads yields an expected signal of $1.43 \mu \mathrm{V} / \mathrm{ML}$ [3]. Hence, we find the theoretically expected value $-S(c, Q)=1.43 \mu \mathrm{V} / \mathrm{ML} \times 9.9 \cdot 10^{-5} \mathrm{ML} / \mathrm{s}=0.14 \mathrm{nV} / \mathrm{s}$. The higher observed value can be due to beads agglomerating into clusters that sediment faster and the influence of other forces (magnetic- or electrostatic forces) attracting the beads to the edges of the sensors, where they are more sensitive. An analysis for a general fluid flow in the channel shows that the amount of beads hitting the bottom of the channel due to gravitational sedimentation is independent of $Q$. Thus, if the beads are assumed to stick to where they land and gravitational sedimentation is the only mechanism at play, $S(c, Q)$ is expected to be independent of $Q$. Realistically, a higher value of $Q$ should make it less probable for beads to remain on the surface on which they land and a reduction of the numerical value of $S(c, Q)$ is expected for increasing $Q$.
This is contrary to the observations in Fig. 6, which show an increase of the numerical value of $S(c, Q)$ upon an increase of $Q$. This observation must be explained by another bead capture mechanism. The field due to the bias current in the sensor and the magnetostatic field from the edges normal to the $x$-direction of the active ferromagnetic sensor layer creates a significant magnetic field gradient near the sensor. These fields will result in a localized force attracting beads in the vicinity of the sensor. Evidence of beads being attracted to the sensor edges was observed after the measurement as seen in Fig. 8.

The amount of beads entering the magnetostatic field region is proportional to $Q$ and this contribution will dominate the capture of beads at high $Q$. This explanation is qualitatively consistent with the observations in Fig. 6. A full quantitative model of the bead sedimentation is too complex for the scope of this paper.

Washing with water removes beads from the sensor even at $Q$ as low as $0.05 \cdot 10^{-9} \mathrm{~m}^{3} / \mathrm{s}$ as shown in Fig. 7. Thus, when a bead suspension is injected there could be a significant removal of beads from the sensor at most values of $Q$. Given time, steady state should be reached where sedimentation and removal of beads balances. The higher the $Q$, the lower the amount of beads on the sensor at steady state should be, and the faster this steady state will be reached. This is observed in the measurements in Fig. 5.

\section{CONCLUSION}

In this study the effect of concentration and flow rate on bead capture on sensors was studied. It was found that the sensor signal from the beads scaled directly with the bead concentration. At low flow rates the bead capture rate was slightly higher than expected from gravitational sedimentation of single beads. It was shown that beads could be completely washed off the sensor surface with a high flow rate of water, but also that low flow rates contributed to the removal of captured beads. Increasing flow rates of the bead suspension enhanced the bead capture rate. This was attributed to bead capture in the areas with high gradient of the magnetic field strength found at the sensor edges. If a homogenous bead distribution is wanted it is thus important to use a low $Q$.

\section{ACKNOWLEDGMENT}

Financial support from the EU 6th Framework Programme BIODIAGNOSTICS project (Contract No. 017002) and The Danish Research Council for Technology and Production Sciences (Grant No. 274-06-0454) is acknowledged.

\section{REFERENCES}

[1] C. D. Damsgaard, S. C. Freitas, P. P. Freitas, and M. F. Hansen, "Exchange-biased planar hall effect sensor optimized for biosensor applications," Journal of Applied Physics, vol. 103, p. 07A302, 2008.

[2] L. Ejsing, M. F. Hansen, A. K. Menon, H. A. Ferreira, D. L. Graham, and P. P. Freitas, "Planar hall effect sensor for magnetic micro- and nanobead detection," Applied Physics Letters, vol. 84, p. 4729, 2004.

[3] T. B. G. Hansen, C. D. Damsgaard, and M. F. Hansen, Unpublished results.

[4] V. Gehanno, P. P. Freitas, A. Veloso, J. Ferreira, B. Almeida, J. B. Sousa, A. Kling, J. C. Soares, and M. F. da Silva, "Ion beam deposition of mnir spin valves," IEEE TRANSACTIONS ON MAGNETICS, vol. 35, pp. 4361-4367, 1999. 